\title{
La smart city: versión 2.0 del municipio
}

\author{
Moisés Barrio Andrés \\ Letrado del Consejo de Estado \\ Doctor en Derecho \\ moises.barrio@consejo-estado.es
}

\section{INTRODUCCIÓN}

La configuración clásica del municipio está siendo objeto de un proceso de actualización para dar paso a la smart city, es decir, para convertir el municipio en más eficiente, sostenible, participativo y con una mayor calidad de vida para sus habitantes gracias a la utilización de las tecnologías de información y comunicación (TIC), y todo ello con el objetivo estructural de hallar soluciones innovadoras a los problemas y retos que tienen que afrontar las ciudades de hoy en día.

La noción smart city posee múltiples implicaciones, desde las infraestructuras y las comunicaciones, pasando por la gestión de residuos, la energía o la provisión de los servicios públicos municipales. Aunque se ha asociado con objetivos como la sostenibilidad, la mejora y la calidad de los servicios públicos, la participación ciudadana, o la eficiencia y la optimización de recursos, lo cierto es que existe un amplio debate doctrinal sobre qué cualifica realmente como «inteligente» a la ciudad y cuáles son los servicios, prioridades y finalidades que tiene que cumplir.

Los proyectos de ciudades inteligentes se caracterizan por un destacado elemento de innovación tecnológica que permite una gestión más avanzada y eficiente de los servicios municipales. La aplicación de las TIC en la gestión de las smart cities plantea varios retos más allá de su componente estrictamente técnico. La tecnología y el uso de datos masivos (big data) brindan nuevas oportunidades de gestión y participación de los ciudadanos en los asuntos municipales, pero también nuevos espacios que precisan de regulación (por ejemplo, los límites de la privacidad, la frontera entre lo público y lo privado, las desigualdades de acceso al mundo digital', etc.). Por otro lado, la tecnología erige un nuevo ecosistema entre los distintos actores urbanos, especialmente entre el ciudadano y la Administración, que altera los modelos de gestión y organización tradicionales y da lugar a nuevas formas de articular la vida social y política.

De ahí la necesidad de regular jurídicamente este cambio de paradigma en la organización municipal, lo cual requiere en primer lugar analizar el concepto de ciudad inteligente.

\section{CONCEPTO Y EVOLUCIÓN}

La primera dificultad en la materia aparece ya a la hora de esclarecer el propio concepto de smart city, pues sus significados han evolucionado -y siguen transformándose- con mucha rapidez en poco tiempo.

Existe, además, un inconveniente añadido, ya que cada dirección doctrinal ha propuesto su propia definición, aunque todas ellas presentan un elemento común: un alto contenido utópico y especulativo sobre cómo las nuevas tecnologías podrían transformar la ciudad. En este sentido, hay que tener en cuenta cómo el contexto material de la noción ha sido prácticamente inexistente en sus inicios -la smart city existía más como idea que como realidad-, lo cual favoreció ese carácter futurista y utópico. De este modo, como han destacado GOLDSMITH y CRAWFORD², la smart city ha venido siendo "un puro concepto de expectativa», pues su evolución ha estado marcada más por las expectativas de futuro levantadas que por su papel como concepto vinculado a la realidad.

1 Lo hemos analizado en BARRIO ANDRÉS, Moisés: “El acceso a Internet como elemento cardinal del servicio universal de telecomunicaciones", en PAREJO ALFONSO, Luciano; VIDA FERNÁNDEZ, José y ESTELLA DE NORIEGA, Antonio (coords.): Los retos del Estado y la Administración en el siglo xxi. Libro homenaje al profesor Tomás de la Quadra-Salcedo Fernández del Castillo. Editorial Tirant Lo Blanch, Valencia, 2017.

2 GOLDSMITH, Stephen y CRAWFORD, Susan: The Responsive City: engaging communities through data-smart governance. Editorial Jossey-Bass, Nueva Jersey, 2014, pág. 3 y ss. 
En todo caso, los diversos autores han venido edificando el concepto en torno a dos elementos: uno, relativo al desarrollo sostenible, y otro, referido al papel transformador de las TIC.

El primer elemento tiene sus orígenes en el movimiento que aparece en Estados Unidos en la década de 1980 denominado New Urbanism. Esta corriente promueve un nuevo tipo de planificación urbanística centrada en la mejora del entorno urbano y la calidad de vida de las personas que lo habitan. Ciudades más compactas, con amplios espacios verdes que faciliten las actividades comunitarias, un diseño que permita una movilidad menos dependiente de los combustibles fósiles y, en definitiva, una mayor eficiencia energética son el núcleo de las propuestas de esta dirección acerca de lo que vendría a significar y materializar una ciudad inteligente³.

El segundo elemento está referido a la propia infraestructura tecnológica subyacente en las smart cities. En efecto, las TIC se convierten en una herramienta esencial, ya que las misiones de sostenibilidad y eficiencia encomendadas sólo pueden alcanzarse a partir del análisis y la explotación de la información que se genera como consecuencia del uso intensivo de medios informáticos y electrónicos y, en concreto, de los datos asociados a la prestación y utilización de los servicios, espacios e infraestructuras de titularidad municipal que ahora propician el big data, la computación en la nube (cloud computing) y el Internet de las cosas (IOT)

Tampoco han faltado autores, como HOLLANDS 5 , que conceptúan la smart city como una versión high-tech de la ciudad emprendedora. Esta dirección doctrinal propugna una mayor influencia de actores del sector privado en la definición del modelo de ciudad, concretamente de las grandes multinacionales del sector tecnológico y energético, como IBM, Cisco, Siemens, Schneider Electric o General Electric, entre otras. Estas empresas tendrían una relación directa con el gobierno municipal y su auxilio se plasmaría mediante los contratos de colaboración entre el sector público y el sector privado. Incluso la Unión Europea ha contribuido al auge de esta orientación, sobre todo a través del programa Horizonte $2020^{6}$, donde potencia los temas de innovación tecnológica urbana y agenda digital, además del desarrollo de la sostenibilidad.

A la postre, la experiencia derivada de las primeras políticas públicas que han implementado smart cities ha desembocado en una convergencia de ambos elementos como han estudiado GRECO y BENCARDINO?

Aparecen así múltiples proyectos que se autodenominan smart y que asumen los diversos elementos antes mencionados, acuñándose definiciones integradoras ante la necesidad de encontrar indicadores objetivos con los que diseñar los proyectos y medir el éxito de las implementaciones existentes. Quizá el ejemplo más característico de esta dirección sea el proyecto del Centre of Regional Science de la Universidad Tecnológica de Viena, titulado «European smart cities», que propone una definición operativa basada en seis ejes o dimensiones: smart economy ${ }^{8}$, smart mobility ${ }^{9}$, smart environment, smart people ${ }^{10}$, smart living ${ }^{11}$ y smart governance ${ }^{12}$. Estas dimensiones acoplan los elementos tradicionales del desarrollo sostenible e innovación tecnológica, y permiten que ciudades pequeñas o medianas puedan ser también conceptuadas como «smart».

En definitiva, la smart city podría ser definida como:

Aquel municipio en el cual las inversiones contribuyen al desarrollo económico sostenible y a una alta calidad de vida con una adecuada gestión de los recursos naturales mediante un gobierno abierto.

Es de notar que, con esta definición, lo esencial no es tanto que el municipio tenga una mínima población municipal, sino que su plan de smart city se proyecte sobre las dimensiones indicadas: economía sostenible, mo-

3 Por todos, THORNS, David: The transformation of cities. Editorial Palgrave, Nueva York, 2002.

4 Por todos, CARAGLIU, Andrea; DEL BO, Clara y NJKAMP, Peter: "Smart cities in Europe", en Central European Conference in Regional Science, CERS, 2009.

5 HOLLANDS, Robert: “Will the real smart city please stand up?", en City, núm. 12, 2009.

6 Vid. https://ec.europa.eu/programmes/horizon2020/

7 GRECO, Ilaria y BENCARDINO, Massimiliano: "Smart Communities. Social innovation at the service of the smart cities", en Tema: Journal of Land Use, Mobility and Environment, 2014, pág. 40 y ss.

8 Así, en la smart economy se incluyen como factores el espíritu innovador, el emprendimiento, la productividad, la flexibilidad del mercado laboral, la internacionalización y la capacidad de transformación.

9 En cuanto a la movilidad (smart mobility) y el medio ambiente (smart environment), son bien conocidas las ventajas y las oportunidades que ofrecen para mejorar las ciudades y para el desarrollo de empresas relacionadas con ambos conceptos.

10 El concepto de smart people implica que las personas tienen buena educación digital, capacidad de aprendizaje, y que cuentan además con competencias de pluralidad, diversidad, flexibilidad y creatividad.

11 Esta dimensión comprende aquellas iniciativas destinadas a lograr que los hábitos de consumo y el comportamiento de los ciudadanos sean saludables y seguros, en el marco de una ciudad que les ofrezca una gran oferta cultural y altos niveles de cohesión social.

12 Por último, bajo el buen gobierno (smart governance) se incluirían aspectos como la participación en los procesos de decisión, el nivel y calidad de los servicios públicos, la transparencia y el contar con una estrategia para la ciudad. 
vilidad, medio ambiente y gobierno abierto. Evidentemente la asunción de las inversiones necesarias para dotar al municipio de la tupida red de comunicaciones que incorpore a los diversos objetos y sensores interconectados -que, de manera automatizada y permanente, proporcionan innumerables datos para la mejor y más eficiente prestación de los servicios- va a requerir una mínima envergadura, que en las experiencias comparadas ${ }^{13}$ se viene concretando en una horquilla mínima de 20.000 a 50.000 habitantes, si bien el progresivo abaratamiento de los dispositivos electrónicos determinará probablemente una reducción del umbral en un futuro no muy lejano. Por tanto, no sería necesario en nuestra opinión que el municipio cumpla con los requisitos del artículo 121 de la Ley 7/1985, de 2 de abril, Reguladora de las Bases del Régimen Local (LBRL en lo sucesivo), esto es, el relativo al régimen de organización de los municipios de gran población.

Nuestra doctrina iuspublicista, por su parte, apenas ha dedicado estudios monográficos a esta figura. Por todos, cabe destacar los estudios de VALERO TORRIJOS ${ }^{14}$ acerca de las implicaciones en la protección de datos, o el trabajo de GARCÍA RUBIO 15, que define la ciudad inteligente como "un fenómeno de implantación en el entorno urbano de una serie de avances tecnológicos y disposiciones técnicas, que permiten una mejor relación con el entorno de la ciudadanía", y que implicaría cuatro ejes: a) un espacio urbano; b) un sistema de infraestructuras; c) un complejo de redes de plataformas inteligentes y; d) una ciudadanía que ejerza diferentes grados de participación política.

Las últimas orientaciones, como ha analizado entre nosotros FERNÁNDEZ BARBUDO ${ }^{16}$, hacen hincapié en el carácter «abierto» y de «laboratorio» de la ciudad inteligente.

Así, más que un objetivo concreto, ahora comienza a adjetivarse este concepto desde la noción de living $l a b^{17}$ : la ciudad se convierte en un gran prototipo, nunca acabado, por lo que cobra importancia la reflexión sobre la interoperabilidad de los distintos sistemas que usan las ciudades inteligentes (open data). La idea de que un Ayuntamiento pueda desarrollar un único programa para convertir la ciudad en inteligente ha dado paso a una visión centrada en múltiples proyectos ${ }^{18} \mathrm{y}$ a la búsqueda de plataformas que permitan el desarrollo continuado de la smart city. A esto hay que sumar la incipiente aparición de diversos proyectos ciudadanos que se reclaman también parte de la ciudad: gracias al desarrollo de la progresiva miniaturización de la electrónica y el abaratamiento de los equipos -los proyectos Arduino, Raspberry Pi y el Internet de las Cosas (IoT) son claves en este sentido- ha emergido un perfil de ciudadano que no es sólo receptor de servicios, sino que también es parte activa en el desarrollo de proyectos municipales. En esta dirección, cabe citar por ejemplo la creación de plataformas de intercambio de conocimiento y recursos P2P entre ciudadanos y también con las instituciones. La ciudad de Seúl ${ }^{19}$ ha sido pionera en este aspecto al desplegar en 2012 un plan para desarrollar proyectos compartidos con diferentes actores.

Ahora bien, y como ha resaltado la doctrina ${ }^{20}$, es imprescindible llevar a cabo un tratamiento exhaustivo de los principales indicadores de una smart city con el fin de evitar el empleo erróneo de este término, que ha sido acuñado de forma impropia por algunas ciudades con el fin de hacerse más atractivas desde el punto de vista turístico o industrial. Siguiendo en este punto a HOLLANDS, debemos notar, en primer lugar, que el concepto de smart city puede conducir a minusvalorar los posibles efectivos negativos del desarrollo de las nuevas tecnologías y de las infraestructuras de red necesarias para que una ciudad sea inteligente; en segundo lugar, la insistencia en su interés estratégico puede dar lugar que se pasen por alto otros caminos alternativos para logar asimismo un prometedor desarrollo urbano ${ }^{21}$.

En definitiva, la noción de smart city, lejos de ser un concepto cerrado, constituye una noción abierta en permanente evolución. Visto su concepto, a continuación debemos dirigir nuestra atención a su régimen jurídico.

13 En España, en torno al 70\% de la población reside en áreas con más de 50.000 habitantes, según los datos del Instituto Nacional de Estadística facilitados con fecha 1 de enero de 2016 (Padrón. Población por municipios). Vid. http://www.ine.es/jaxi/menu.do?type=pcaxis\&path $=\% 2 \mathrm{Ft} 20 \% 2 \mathrm{Fe} 260 \&$ file=inebase .

14 VALERO TORRIJOS, Julián: "Sostenibilidad y gestión de la información en las ciudades inteligentes (smart cities): apuntes para un debate desde la perspectiva jurídica", en BUSTILLO BOLADO, Roberto (coord.): Desarrollo sostenible: análisis jurisprudencial y de políticas públicas. Editorial Aranzadi, Navarra, 2014, y VALERO TORRIJOS, Julián: Derecho, innovación y Administración electrónica. Editorial Global Law Press, Sevilla, 2013.

15 GARCÍA RUBIO, Fernando: "Planeamiento, movilidad y sostenibilidad urbana. Un análisis desde las Smart cities", en Práctica Urbanística, núm. 131, 2014.

16 FERNÁNDEZ BARBUDO, Carlos: “El papel de la Smart City en el concepto de privacidad”, en BALCELLS PADULLÉS, Joan (coord.): Regulating Smart Cities. Editorial Huygens, Barcelona, 2015, pág. 96.

17 SCHAFFERS, Hans (coord.): Smart Cities as Innovation Ecosystems sustained by the Future Internet. FIREBALL White Paper, 2012, pág. 5 y ss.

18 GRECO y BENCARDINO, op. cit., pág. 45.

19 Vid. http://english.seoul.go.kr/policy-information/key-policies/city-initiatives/1-sharing-city/.

20 RIVERO ORTEGA, Ricardo y MERINO ESTRADA, Valentín: Innovación y gobiernos locales: estrategias innovadoras de ayuntamientos y diputaciones provinciales en un contexto de crisis. Editorial Instituto Nacional de Administración Pública, Madrid, 2014, pág. 94.

21 Op. cit., pág. 303 y ss. 


\section{RÉGIMEN JURÍDICO}

España cuenta ya en 2016 con diversas realizaciones de smart cities agrupadas ${ }^{22}$ en torno a la Red Española de Ciudades Inteligentes $(\mathrm{RECl})^{23}$, que tiene por objeto intercambiar experiencias y trabajar conjuntamente para desarrollar un modelo de gestión sostenible y mejorar la calidad de vida de los ciudadanos, incidiendo en aspectos como el ahorro energético, la movilidad sostenible, la Administración electrónica, la atención a las personas o la seguridad.

Recientemente, en marzo de 2015 se ha dado un paso más en la consecución de estos objetivos mediante la

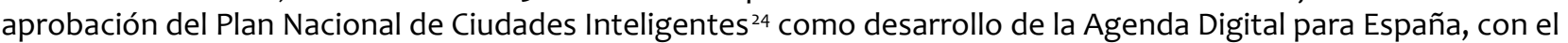
que se pretenden, entre otras, las siguientes metas: a) aumentar la aportación de las TIC al PIB del sector industrial; b) mejorar la eficacia y eficiencia de las Entidades Locales en la prestación de los servicios públicos a través del uso de las TIC; c) avanzar en la gobernanza del sistema de Ciudades Inteligentes; y d) impulsar la estandarización, regulación y normativa de las smart cities.

Por el contrario, no existe un grupo normativo sectorial que regule la intrincada problemática de esta figura, siendo las previsiones de la LBRL y de la Ley 37/2007, de 16 de noviembre, sobre reutilización de la información del sector público (abreviadamente LRISP) notablemente insuficientes como consecuencia de las complicadas relaciones jurídicas subyacentes. En efecto, todo proyecto de smart city va cimentado sobre una pluralidad de servicios de diversa naturaleza que se prestan por sujetos distintos y, lo que resulta incluso más relevante desde la perspectiva jurídica, con arreglo a normas dispares.

En primer lugar, por una parte confluyen servicios de carácter privado, en ocasiones procedentes de sectores liberalizados, tal y como puede suceder singularmente con la provisión de servicios de telecomunicaciones o el suministro de energía eléctrica, lo que conlleva que una pluralidad de prestadores ofrezcan tales servicios y, en consecuencia, los datos que puedan proporcionar sean necesariamente fragmentarios, circunstancia que supone un obstáculo desde la necesidad de un tratamiento agregado de los datos que exige una smart city. Dada la naturaleza privada de las relaciones jurídicas subyacentes, la libre competencia existente en estos mercados, así como la ausencia de título competencial adecuado por parte de la Administración municipal, las principales dificultades en cuanto a la explotación integrada de los datos derivarán, en gran medida, de los intereses contrarios que pueden tener varias empresas privadas que operan en el mismo ámbito territorial en un mercado liberalizado, de manera que se van a producir importantes reticencias para compartir información entre ellas y con terceros, sin que, por su parte, la Administración local tenga posibilidad alguna de obligarles a integrar los datos correspondientes a dichos servicios en la medida que escapan de su ámbito competencial propio.

No sucede lo mismo, en cambio, por lo que respecta a los servicios públicos cuya titularidad corresponde a los municipios. En efecto, al margen de aquellos supuestos en que la propia entidad local los preste a través de algunos de los modos directos a los que se refiere el artículo 86.2 de la LBRL, en los que la capacidad de control municipal sobre la gestión de los datos que se generen resulta indubitada, en el caso de que la gestión se lleve a cabo bajo alguna de las modalidades indirectas nos encontraríamos ante un supuesto que se tiene que prestar mediante las distintas formas previstas para el contrato de gestión de servicios públicos en el texto refundido de la Ley de Contratos del Sector Público (art. 85.2.B) LBRL). En consecuencia, será necesario precisar minuciosamente el concreto objeto de las prestaciones inherentes a la relación contractual de cada servicio para, de este modo, determinar el régimen jurídico aplicable y, en concreto, regular con detalle la gestión y tratamiento de los datos.

En segundo lugar, la intervención del sector privado también se proyecta más allá de los propios servicios públicos, ya que la entrada en acción de las interconexiones y gestión avanzada de los datos que se realiza en el seno de la smart city -gracias al calor tanto del big data como del Internet de las cosas- determinan la necesaria participación de otros sujetos privados. En efecto, como consecuencia del contexto tecnológico antes indicado, entran en juego nuevos prestadores de servicios que facilitan el acceso a las redes que interconectan los dispositivos y sensores como, asimismo, a los servicios de la sociedad de la información necesarios (v. gr., empresas que

22 A fecha de escribir estas líneas, RECl está formada por 65 ciudades: A Coruña, Albacete, Alcalá de Henares, Alcobendas, Alcorcón, Alicante, Almería, Alzira, Aranjuez, Arganda del Rey, Ávila, Badajoz, Barcelona, Burgos, Cáceres, Castellón, Ciudad Real, Córdoba, Guadalajara, El Puerto de Santa María, Elche, Fuengirola, Getafe, Gijón, Jaén, L’Hospitalet de Llobregat, Huelva, Las Palmas de Gran Canaria, Logroño, Lugo, Huesca, Madrid, Majadahonda, Málaga, Marbella, Mérida, Molina de Segura, Móstoles, Motril, Murcia, Oviedo, Palencia, Palma de Mallorca, Pamplona, Paterna, Ponferrada, Pozuelo de Alarcón, Rivas Vaciamadrid, Sabadell, Salamanca, San Cristóbal de La Laguna, Sant Cugat, Santa Cruz de Tenerife, Santander, Santiago de Compostela, Segovia, Sevilla, Tarragona, Toledo, Torrejón de Ardoz, Torrent, Valencia, Valladolid, Vitoria-Gasteiz y Zaragoza.

23 Impulsada en junio de 2011 por el actual Ministro de Fomento, Íñigo de la Serna Hernáiz, en su anterior condición de Alcalde de Santander.

24 Vid. http://www.agendadigital.gob.es/planes-actuaciones/Bibliotecaciudadesinteligentes/Detalle\%20del\%20Plan/Plan_Nacional_de_Ciudades_Inteligentes_v2.pdf. 
proporcionan servicios de almacenamiento en la nube, aplicaciones para smart phones dirigidas a la ciudadanía, etc.). De este modo, aun cuando su participación no resulte estrictamente necesaria para la prestación de los servicios públicos desde la clásica óptica del servicio público, el fuerte componente tecnológico en el que se sustentan las ciudades inteligentes les confiere un papel protagonista dada la complejidad técnica de este tipo de iniciativas.

De este modo, y ante la falta de regulación por el momento, los pliegos contractuales se convierten en un instrumento estructural para determinar el régimen jurídico en el cual se desplegará y ejecutará la smart city, ya que la fuerte carga de innovación tecnológica que requiere no parece que pueda conseguirse exclusivamente con los medios propios de las entidades públicas implicadas.

Sentado lo anterior, en sede de conclusiones vamos a poner de manifiesto los riesgos más urgentes de atención por parte del legislador de cara a un desarrollo correcto y estable de este tipo de iniciativas, máxime cuando las entidades privadas involucradas reclaman un marco legal más claro, seguro, transparente y favorable a la inversión, propugnando incluso plazos más largos de ejecución de los contratos, agrupación de servicios, contratación por objetivos o, en fin, modelos de compra pública innovadora ${ }^{25}$.

\section{CONCLUSIONES}

El propósito final de una smart city es alcanzar una gestión eficiente en todas las áreas de la ciudad (urbanismo, infraestructuras, transporte, servicios, educación, sanidad, seguridad pública, energía, etc), satisfaciendo a la vez las necesidades de la urbe y de sus ciudadanos. Todo ello debe lograrse en consonancia con los principios de Desarrollo Sostenible expuestos en el denominado Programa $21^{26}$, promovido por Naciones Unidas, y tomando la innovación tecnológica y la cooperación entre agentes económicos y sociales como los principales motores del cambio.

Para ello se sirve, en gran medida, de una gestión avanzada de la información, lo que precisa que el tratamiento de los datos se realice de manera automatizada, inmediata, predictiva y, de este modo, se puedan ofrecer servicios de valor añadido, tanto por lo que concierne a la actividad municipal como a los ciudadanos usuarios de los servicios.

Además, la elevada complejidad no sólo es una característica inherente a los proyectos de smart cities sino que, también y como consecuencia inevitable, se traslada a la inexcusable tarea de determinar e interpretar el marco normativo aplicable. Más aún, como se ha destacado con acierto ${ }^{27}$, la especial complejidad de las normas sobre protección de datos constituye una dificultad adicional, de manera que difícilmente podrán ofrecerse respuestas precisas que no requieran de matices y condicionamientos, en gran medida exigidos por la confluencia de bienes jurídicos potencialmente contrapuestos. Esta dificultad adquiere una especial trascendencia en la medida que las ciudades inteligentes demandan un entorno abierto en el que los datos se encuentren a disposición de los diversos actores en formatos abiertos y compatibles que permitan su reutilización automatizada, ya que es imprescindible un tratamiento útil de los datos para poder ofrecer servicios de valor añadido a los ciudadanos.

Es crucial destacar cómo son múltiples los beneficios que pueden conseguir los diferentes sujetos involucrados en una ciudad inteligente. En primer lugar, la Administración local emplea la información obtenida para hacer más eficaz la oferta de los servicios públicos efectivamente demandados por los ciudadanos, para optimizar la utilización de fondos destinados a subvencionar los servicios públicos locales y para crear sistemas integrados y multimodales. En segundo lugar, las empresas que prestan los servicios públicos se benefician de una distribución más equitativa de los recursos públicos y pueden aprovechar los datos para planificar mejor sus servicios y modelos de negocio. Los usuarios, por último, tienen la posibilidad de acceder a su propia información, de disfrutar de sistemas inteligentes de prestación de servicios públicos más eficaces y, en muchos casos, de utilizar sistemas de pago electrónicos y online. A nivel colectivo, además, los ciudadanos y las administraciones locales tienen acceso a la información en forma agregada, lo que hace más fácil definir estrategias de reducción de la congestión de los servicios, apoyar la planificación urbanística, potenciar los servicios de uso compartido (por ejemplo los servicios de bike sharing o car sharing combinados con el uso de medios públicos de transporte), así como crear nuevos

25 Respecto de la reforma reclamada por el sector privado, vid. SEISDEDOS, Gildo: Smart cities: La transformación digital de las ciudades. Editorial Instituto de Empresa-PwC, Madrid, 2015, pág. 105.

26 Vid. http://www.un.org/spanish/esa/sustdev/agenda21/index.htm.

27 ZIEGLER, Sébastien y SONKO, Kémo: "Privacy Risk Area Assessment Tool for Audio Monitoring - from legal complexity to practical applications", en Journal of International Commercial Law and Technology, vol. 9, núm. 3, 2014, págs. 146 y 147. Entre nosotros, vid. VALERO TORRIJOS, Julián: "Sostenibilidad y gestión de la información en las ciudades inteligentes (smart cities): apuntes para un debate desde la perspectiva jurídica”, en BUSTILLO BOLADO, Roberto (coord.): Desarrollo sostenible: análisis jurisprudencial y de políticas públicas. Editorial Aranzadi, Navarra, 2014, pág. 477 y ss. 
servicios comerciales (por ejemplo, programas de fidelización para los abonados o técnicas publicitarias basadas en la posición en tiempo real del usuario).

Por el contrario, un uso indebido y que no garantice la debida privacidad de los datos puede modificar un contexto centrado en una aplicación positiva de la innovación y transformarlo en un ambiente inteligente perturbador, en el que los gobiernos monitoricen los hábitos y las interacciones sociales de los ciudadanos, las empresas aprovechen la información para desplegar prácticas comerciales incorrectas o que vulneren la libre competencia, enviar publicidad no deseada a los usuarios o realizar análisis de mercado de manera oculta.

A la postre, la bondad de la innovación tecnológica no puede conllevar la disminución de las garantías jurídicas de los usuarios de tales servicios y, en general, de los ciudadanos, en particular por lo que se refiere a la seguridad en el uso de su información personal, que sólo puede ser utilizada para aquellas finalidades lícitas que justifiquen la recogida de los datos.

\section{BIBLIOGRAFÍA}

VV.AA: Smart Cities, Ranking of European medium-sized cities. Final report. Vienna University of Technology, Delft University of Technology, University of Ljubljana. Edición: Centre of Regional Science, Vienna UT, 2007.

BARRIO ANDRÉS, Moisés: "El acceso a Internet como elemento cardinal del servicio universal de telecomunicaciones", en PAREJO ALFONSO, Luciano; VIDA FERNÁNDEZ, José y ESTELLA DE NORIEGA, Antonio (coords.): Los retos del Estado y la Administración en el siglo xxi. Libro homenaje al profesor Tomás de la Quadra-Salcedo Fernández del Castillo. Editorial Tirant Lo Blanch, Valencia, 2017.

CARAGLIU, Andrea; DEL BO, Clara y NJKAMP, Peter: "Smart cities in Europe", en Central European Conference in Regional Science, CERS, 2009.

FERNÁNDEZ BARBUDO, Carlos: "El papel de la Smart City en el concepto de privacidad", en BALCELLS PADULLÉS, Joan (coord.): Regulating Smart Cities. Editorial Huygens, Barcelona, 2015.

GARCÍA RUBIO, Fernando: "Planeamiento, movilidad y sostenibilidad urbana. Un análisis desde las Smart cities", en Práctica Urbanística, núm. 131, 2014.

GOLDSMITH, Stephen y CRAWFORD, Susan: The Responsive City: engaging communities through data-smart governance. Editorial Jossey-Bass, Nueva Jersey, 2014.

GRECO, Ilaria y BENCARDINO, Massimiliano: "Smart Communities. Social innovation at the service of the smart cities", en Tema: Journal of Land Use, Mobility and Environment, 2014.

HOLLANDS, Robert: “Will the real smart city please stand up?", en City, núm. 12, 2009.

QUINTANA LÓPEZ, Tomás (dir.): Urbanismo sostenible: rehabilitación, regeneración y renovación urbanas. Editorial Tirant lo Blanch, Valencia, 2016.

RIVERO ORTEGA, Ricardo y MERINO ESTRADA, Valentín: Innovación y gobiernos locales: estrategias innovadoras de ayuntamientos y diputaciones provinciales en un contexto de crisis. Editorial Instituto Nacional de Administración Pública, Madrid, 2014.

SCHAFFERS, Hans (coord.): Smart Cities as Innovation Ecosystems sustained by the Future Internet. FIREBALL White Paper, 2012.

SEISDEDOS, Gildo: Smart cities: La transformación digital de las ciudades. Editorial Instituto de Empresa-PwC, Madrid, 2015.

THORNS, David: The transformation of cities. Editorial Palgrave, Nueva York, 2002.

VALERO TORRIJOS, Julián: "Sostenibilidad y gestión de la información en las ciudades inteligentes (smart cities): apuntes para un debate desde la perspectiva jurídica", en BUSTILLO BOLADO, Roberto (coord.): Desarrollo sostenible: análisis jurisprudencial y de políticas públicas. Editorial Aranzadi, Navarra, 2014.

VALERO TORRIJOS, Julián: Derecho, innovación y Administración electrónica. Editorial Global Law Press, Sevilla, 2013.

ZIEGLER, Sébastien y SONKO, Kémo: "Privacy Risk Area Assessment Tool for Audio Monitoring-from legal complexity to practical applications", en Journal of International Commercial Law and Technology, vol. 9, núm. 3, 2014. 